\title{
Endovascular aortic repair of a chronic ascending and arch aortic aneurysm
}

Thomas Gandet, MD, Pierre Alric, MD, PhD, Sebastien Bommart, MD, PhD, and

Ludovic Canaud, MD, PhD, Montpellier, France

From the Department of Thoracic and Cardiovascular Surgery, Hospital Arnaud de Villeneuve, Montpellier, France.

Disclosures: Authors have nothing to disclose with regard to commercial support.

Received for publication April 12, 2017; revisions received Aug 15, 2017; accepted for publication Sept 4, 2017; available ahead of print Oct 4, 2017.

Address for reprints: Ludovic Canaud, MD, PhD, Service de Chirurgie Vasculaire et Thoracique, Hôpital A de Villeneuve, 191 Ave Doyen Gaston Giraud, Montpellier 34090, France (E-mail: ludoviccanaud@ hotmail. com).

J Thorac Cardiovasc Surg 2018;155:e79-83

$0022-5223 / \$ 36.00$

Copyright (C) 2017 by The American Association for Thoracic Surgery

https://doi.org/10.1016/j.jtcvs.2017.09.017

$\square$ Video clip is available online.

Although mortalities as great as $60 \%$ have been reported in high-risk patients with type A aortic dissection, open surgical repair remains the first-line therapy. The use of stent grafts in the ascending aorta has been limited to a small number of case reports. ${ }^{1,2}$

The risks of open surgical repair can be prohibitively increased in patients with a hostile chest. Resternotomy can be particularly hazardous in patients with abnormal chest wall anatomy as a consequence of severe kyphoscoliosis, as a complication from previous operations, or secondary to extensive radiotherapy. ${ }^{3}$ Radiation-induced heart disease increases perioperative morbidity and decreases both short- and long-term survivals. ${ }^{4}$ The patient in this case study had previously undergone sternal resection and reconstruction with an omental flap and titanium rib implants, followed by external beam radiotherapy.

We report the use of a "reverse" extra-anatomic debranching procedure to provide a suitable landing zone for thoracic endovascular aortic repair with a homemade single proximal fenestration for the left subclavian artery (LSA) in a patient with aneurysmal degeneration of a dissection of the ascending and arch of the aorta.

\section{CLINICAL SUMMARY}

Our Institutional Ethical Committee approved the approach described here. The patient was a 78-year-old man who was admitted with chest pain and a history of primary osteosarcoma of the sternum 2 years previously,

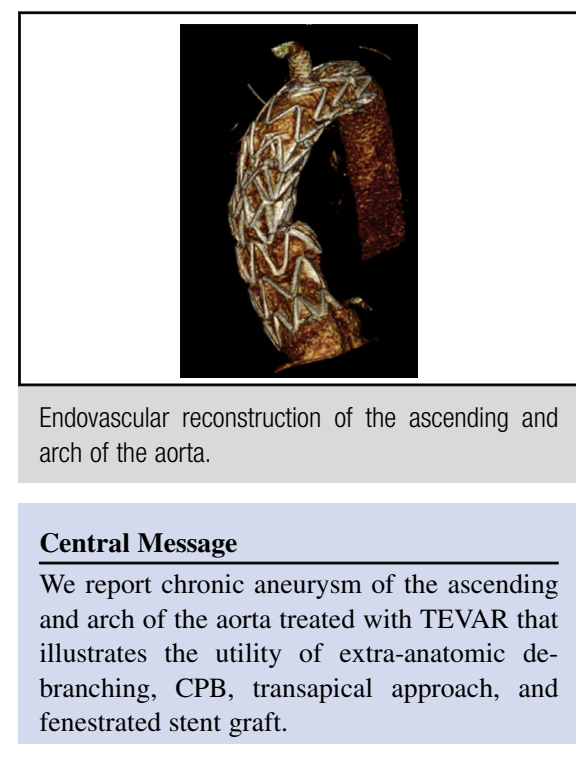

See Editorial Commentary page e85.

which had been treated with sternal resection and reconstruction with omental flap and titanium rib implants followed by external beam radiotherapy (60 Gy). The patient's osteosarcoma was in remission. Computed tomography (CT) demonstrated a large dissecting aneurysm of the ascending and arch of the aorta. In the routine oncologic follow-up CT scan 4 months previously, the ascending aorta had appeared normal. The aneurysm measured $9.7 \mathrm{~cm}$ in anteroposterior diameter (Figures 1 and 2 ). Cardiorespiratory evaluation identified mild aortic regurgitation and radiation cardiomyopathy.

The aneurysm started $6 \mathrm{~mm}$ distally from the ostia of the left coronary artery and ended immediately proximal to the LSA. At 7 days after admission, a "reverse" arch debranching (supra-aortic debranching with outflow through the LSA) procedure was performed. This constituted the following steps: (1) LSA to right axillary artery bypass; (2) sequential reimplantation of the left and right common carotid arteries into the bypass, and (3) proximal ligation of the right and left common carotid artery and right subclavian artery origins (Figure 3). Cerebral monitoring was not used. Clamp times during sequential revascularization of the supra-aortic trunks were less than 10 minutes for each vessel. 


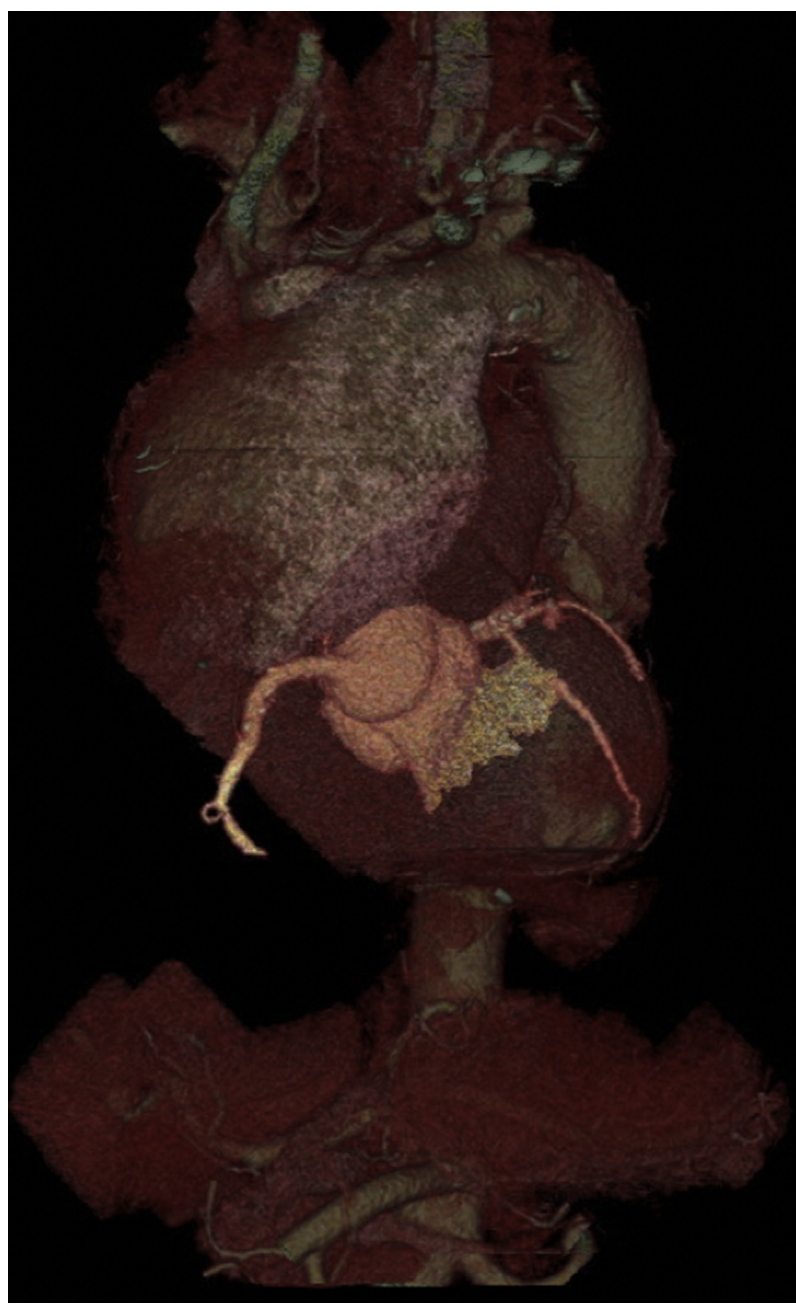

FIGURE 1. Computed tomographic scan 3-dimensional reconstruction of the ascending and arch aortic aneurysm.

Cardiopulmonary bypass (CPB) was established by arterial inflow from the right axillary artery and venous outflow through the femoral vein. Concomitant transapical access was obtained.

A 32- $\times 100-\mathrm{mm}$ Valiant stent graft (Medtronic, Minneapolis, Minn) was selected. A single proximal fenestration was fashioned for the LSA. We have previously reported how to fenestrate thoracic stent grafts. ${ }^{5}$ The stent graft was introduced into the arch through a transapical approach. An angiogram "opening" the aortic arch was performed. To facilitate accurate stent-graft deployment, circulatory pressures were decreased by manipulation of the CPB parameters. After it was ascertained that the fenestration was appropriately oriented facing the LSA origin, the stent graft was partially deployed. From the LSA, a 7F sheath was advanced through the fenestration into the stent graft. Deployment of the thoracic stent graft was completed. A 10-mm balloon-expandable iCAST covered stent (Atrium Medical Corporation, Hudson, NH) was deployed across the fenestration to avoid a type III endoleak.

A second Valiant stent graft (44-100 mm) was deployed, with the proximal landing zone just distal to the coronary ostia. Completion angiogram demonstrated patency of the coronary ostia and of the LSA without endoleak. CPB time was 87 minutes.

The postoperative course was complicated by stroke. The cranial CT of the patient demonstrated multiple lesions involving multiple vascular territories. We believe that this complication could be related to wire manipulation, vessel clamping, endograft deployment, or microemboli during CPB. The patient had no motor deficit (hemianopia and apraxia). A tracheostomy was required for prolonged respiratory support. CT angiography demonstrated patency of the stent grafts and extra-anatomic aortic arch branches with no endoleak (Figures 4 and 5 and Video 1). The patient died in the third postoperative month of pulmonary insufficiency secondary to pneumonia.

\section{DISCUSSION}

The anatomic and physiologic challenges to endovascular therapy of the ascending aorta remain formidable. These include proximal graft fixation close to both the aortic valve and the coronary ostia and a distal landing zone that may impinge on the innominate artery. Another challenge is the hemodynamic forces in the ascending aorta, which resist accurate graft deployment.

This case report illustrates the feasibility of a "reverse" aortic arch debranching procedure to provide a suitable distal landing zone allowing debranching via a cervical approach that avoids aortic clamping. By facilitating manipulation of circulatory pressures partial CPB helped to improve stent positioning during graft deployment.

There were several reasons that we adopted transapical access. First, the relatively short length of the delivery sheath excluded transfemoral delivery to such a proximal landing zone. Second, accurate device control and deployment is hindered with femoral access because of diminished control of torque through tortuous iliac vessels into a $270^{\circ}$ aortic arch.

Significant aortic regurgitation can be caused by the large-bore sheath introduced through the aortic valve, especially for this patient with aortic valve regurgitation. Hemodynamic status (worsening of aortic valve regurgitation as a result of the sheath introduced through the aortic valve) was less of a concern with $\mathrm{CPB}$, which allowed the time for meticulous decision making during the relatively complicated procedures in our case.

Stroke is an increasingly recognized complication after thoracic endovascular aortic repair. The cranial CT of the patient demonstrated multiple embolic 


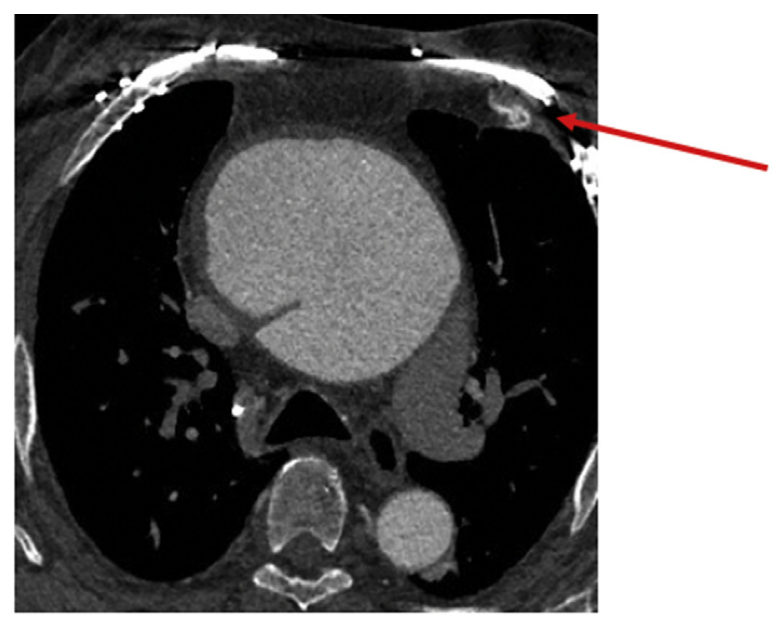

A

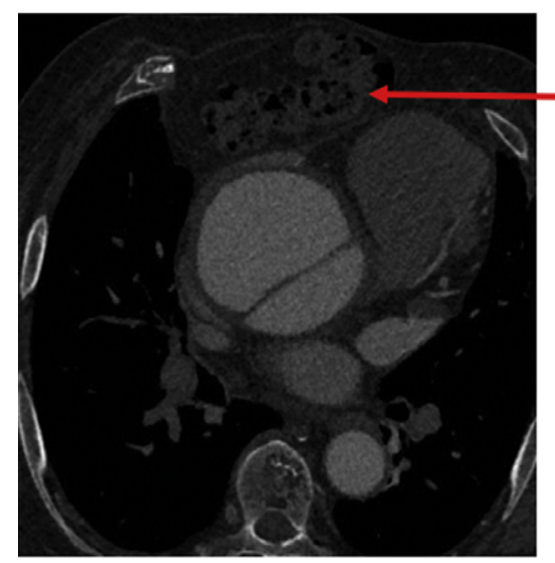

B

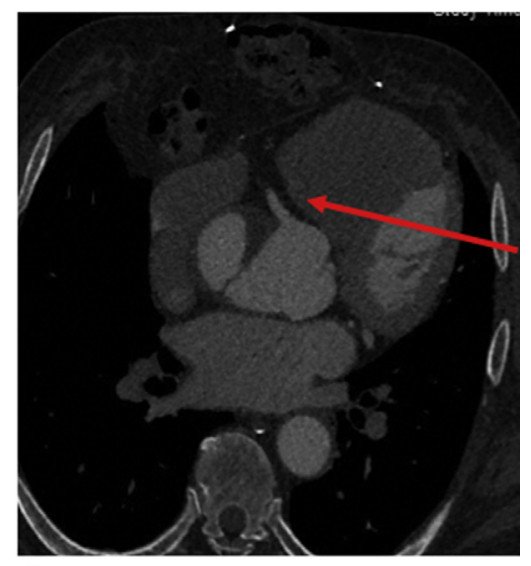

C

FIGURE 2. Preoperative computed tomographic scans show a $10-\mathrm{cm}$ ascending and aortic arch aneurysm. A, Arrow indicates titanium rib implants. B, Arrow indicates the omental flap. C, Arrow indicates the left coronary artery.

infarcts. We believe that this complication was related to wire manipulations, vessel clamping, endograft deployment, or microemboli during CPB.
Atheroembolic complications remain challenging during aortic arch repair, even in cases of endovascular repair. Careful manipulation of central vessels is

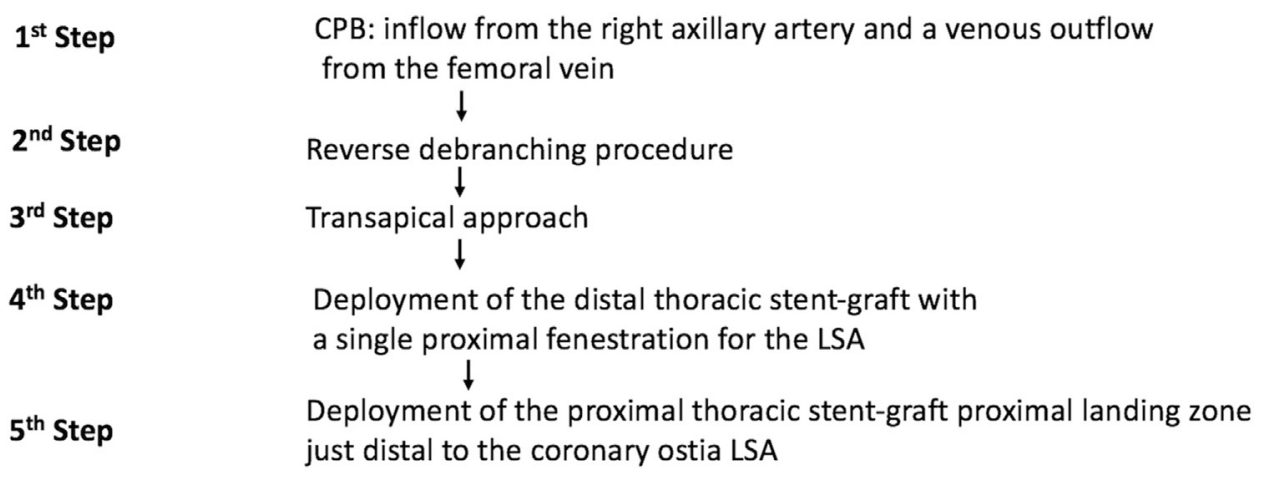

FIGURE 3. Diagram to illustrate the surgical steps undertaken in this procedure. LSA, Left subclavian artery. 


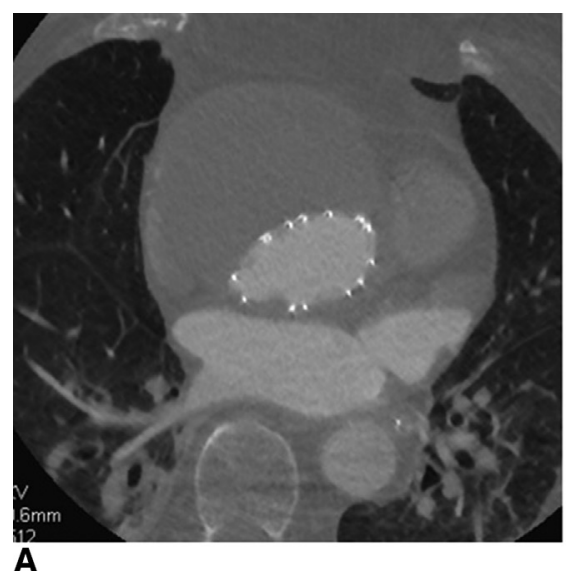

A

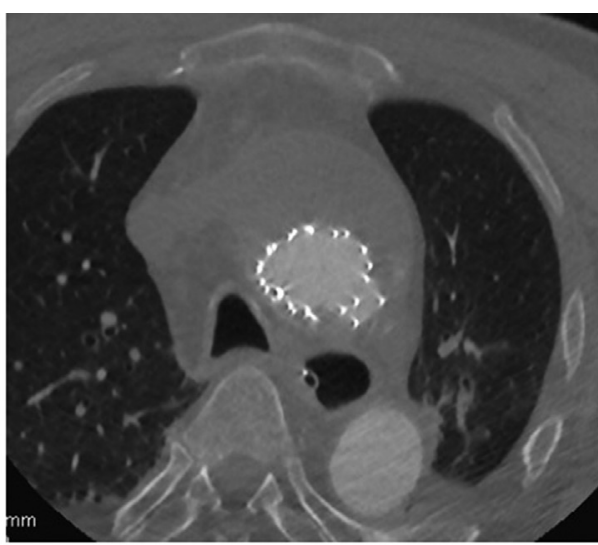

B

FIGURE 4. Axial computed tomographic scans showing the endovascular exclusion of the ascending and aortic arch aneurysm. A, Ascending aorta level. B, Aortic arch level.

mandatory for success, as is keeping crossclamp times short to avoid exceeding the ischemic tolerance of cerebral tissue. Furthermore, the wires in the aortic arch need to be manipulated cautiously to avoid detachment of soft plaque.

\section{CONCLUSIONS}

This report demonstrates a case of chronic aneurysm of the ascending and arch of the aorta treated with thoracic endovascular aortic repair.

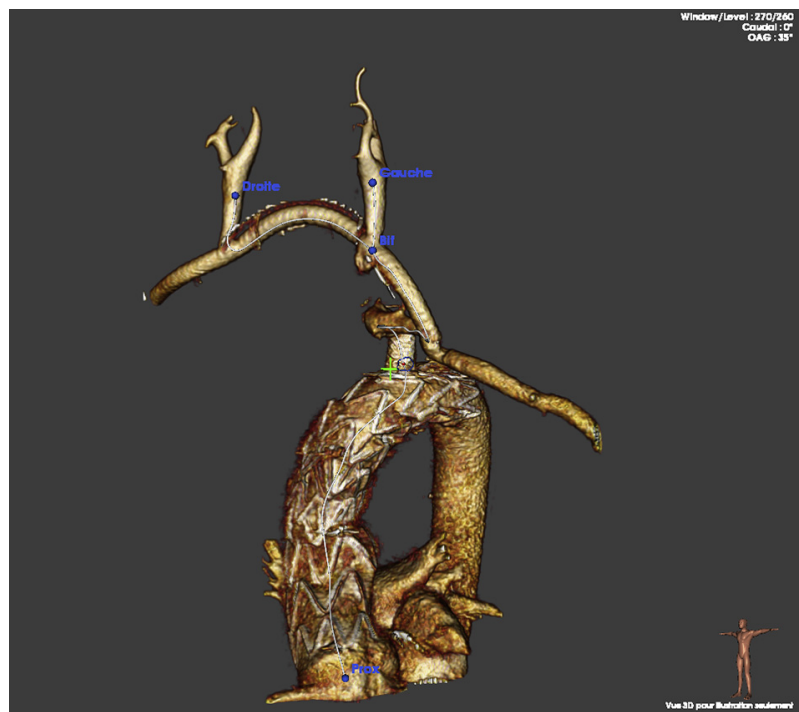

FIGURE 5. Computed tomographic 3-dimensional reconstruction showing "reverse" arch debranching, the distal stent graft with proximal fenestration for the left subclavian artery, and the proximal stent graft with the proximal landing zone just distal to the coronary ostia. Droite, Right; Gauche, left; Bif, bifurcation; Prox, proximal.

\section{References}

1. Ronchey S, Serrao E, Alberti V, Fazzini S, Trimarchi S, Tolenaar JL, et al. Endovascular stenting of the ascending aorta for type A aortic dissections in patients at high risk for open surgery. Eur J Vasc Endovasc Surg. 2013;45: 475-80.

2. Nienaber CA, Sakalihasan N, Clough RE, Aboukoura M, Mancuso E, Yeh JS, et al. Thoracic endovascular aortic repair (TEVAR) in proximal (type A) aortic dissection: Ready for a broader application? J Thorac Cardiovasc Surg. 2017; 153:S3-11.

3. Kappetein AP, Head SJ, Généreux P, Piazza N, van Mieghem NM, Blackstone EH, et al; Valve Academic Research Consortium-2. Updated standardized endpoint definitions for transcatheter aortic valve implantation: the Valve Academic Research Consortium-2 consensus document. J Thorac Cardiovasc Surg. 2013;145:6-23.

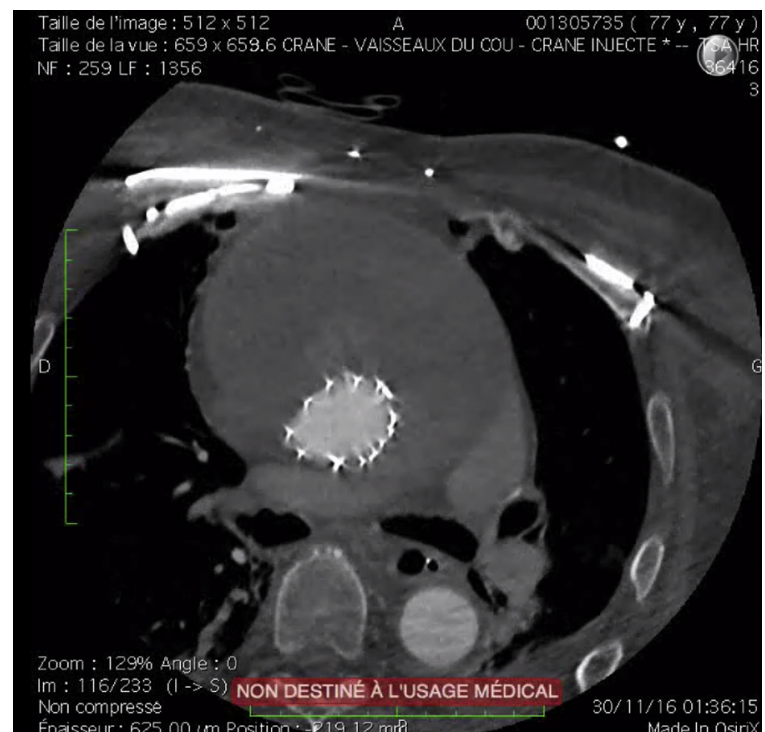

VIDEO 1. Axial computed tomographic scan showing the endovascular exclusion of the ascending and aortic arch aneurysm. Video available at: http://www.jtcvsonline.org/article/S0022-5223(17)31920-7/fulltext. 
4. Chang AS, Smedira NG, Chang CL, Benavides MM, Myhre U, Feng J, et al. Cardiac surgery after mediastinal radiation: extent of exposure influences outcome. J Thorac Cardiovasc Surg. 2007;133:404-13.
5. Canaud L, Baba T, Gandet T, Narayama K, Ozdemir BA, Shibata T, et al Physician modified thoracic stent-grafts for the treatment of aortic arch lesions. J Endovasc Ther. 2017;24:542-8. 